\title{
Development of microbial-fortified rice straw compost to improve plant growth, productivity, soil health, and rice blast disease management of aerobic rice
}

\begin{abstract}
In aerobic rice cultivation systems, compost mulching and incorporation are important to rehabilitate the soil. Microbial-fortified compost is increasingly accepted as a safe approach in agro-waste management to recycling of crop residuals in agriculture soil and also to promote growth and suppress disease. This study aims to examine the stability and viability of the selected plant growth-promoting microorganisms (PGPM) in rice straw compost (RSC) over incubation period and its bio-efficacy in promoting rice (Oryza sativa) plant growth, productivity, soil health, and controlling of Pyricularia oryzae in aerobic cultivation conditions. Six selected PGPM: Pseudomonas aeruginosa (UPMP1), Corynebacterium agropyri (UPMP7), Enterobacter gergoviae (UPMP9), Bacillus amyloliquefaciens (UPMS3), Trichoderma harzianum (UPMT1), and Trichoderma virens (UPMT2) were used as a consortium of microbial inoculants to develop the microbial-fortified rice straw compost (MRSC). The MRSC was incorporated into mineral soil used for aerobic rice cultivation and its bio-efficacy was evaluated at harvest. The viability of Trichoderma spp. found stabilized at $6.78 \mathrm{i} 6.00 \mathrm{log} \mathrm{cfu} / \mathrm{g}$ and declined for all the bacterial isolates. At harvest, soil amended with MRSC significantly increased in plant height, leaf area index, 1000 grain weight, and productivity. The MRSC amended plots had significant low in rice blast disease severity with area under disease progress curve (AUDCP) of 748.22 unit/square, as compared to control (1782.67 unit/square). The physicochemical and microbiological properties of soil amended with MRSC were improved at harvest. The application of MRSC has potential to improve plant growth, productivity, rice blast disease management, and soil health of rice under aerobic cultivation systems.
\end{abstract}

Keyword: Plant growth-promoting microorganisms; Rice straw compost; Microbial-fortified rice straw compost; Aerobic rice 